\title{
Enterovirus 71 infection impairs the reproductive capacity of female mice
}

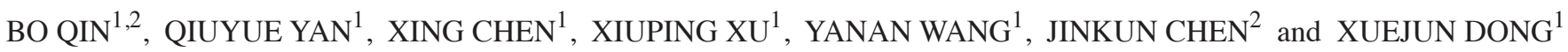 \\ ${ }^{1}$ Clinical Laboratory Center, Shaoxing People's Hospital, Shaoxing Hospital of Zhejiang University; \\ ${ }^{2}$ Shaoxing Centre for Disease Control and Prevention, Shaoxing, Zhejiang 312000, P.R. China
}

Received February 22, 2016; Accepted February 24, 2017

DOI: 10.3892/etm.2017.4499

\begin{abstract}
Enterovirus 71 (EV71) is a major cause of hand, foot and mouth disease (HFMD); however, no clinically approved vaccine or antiviral treatment is currently available for EV71 infection. In the present study, a murine model of EV71 infection was constructed. The clinical isolates of EV71 were amplified in Vero cells and used to challenge adult mice via hydrodynamic injection (HI) and intraperitoneal injection (IP). Following two challenges, $>50 \%$ of the mice succumbed to EV71 infection. Surviving female mice were identified to have impaired fertility and their litter sizes were significantly decreased compared with the control group. The antibody against EV71-VP1 persisted in the sera of female mice at a high titer for $>2$ years after challenge. The maternal antibody in the offspring sera also persisted for $\sim 1$ year and disappeared after $\sim 2$ years. Results from the present study suggest that a high titer of active EV71 was able to impair the reproductivity of adult female mice, and that high levels of maternal antibody persisted in the offspring and protected postnatal mice from EV71-induced mortality. The promising antigenicity, immunogenicity and reactogenicity of EV71 suggests that it a potential vaccine target that may be beneficial to the control of HFMD, through immunizing infants and women of reproductive age.
\end{abstract}

\section{Introduction}

Enterovirus (EV) 71 is a member of the picornaviridae family of viruses that contain single-stranded positive-sense RNA (1), which primarily infects children $<5$ years old (2). Infants with EV71 infection develop sores on their hands, feet, buttocks and mouth; therefore, the infection is referred to as hand, foot and mouth disease (HFMD) (3). Several types of enterovirus have

Correspondence to: Professor Xuejun Dong, Clinical Laboratory Center, Shaoxing People's Hospital, Shaoxing Hospital of Zhejiang University, 568 Zhongxing North Road, Shaoxing, Zhejiang 312000, P.R. China

E-mail: qinbo0809@hotmail.com

Key words: enterovirus 71, immunogenicity, maternal antibody, hydrodynamic injection, immunological stress been indicated to cause HFMD in children; however, EV71 is commonly associated with severe complications of the nervous system in infants with HFMD and is therefore considered to be a major virulent pathogen and cause of mortalities in HFMD (4). Previous studies have indicated that a total of 488,955 HFMD cases and 126 HFMD-associated mortalities were reported in 2008 in China, of which EV71 infection was the primary cause (5). EV71 is a non-enveloped virus with a single-stranded positive-sense RNA genome that is $\sim 7.5 \mathrm{~kb}$ in size and belongs to the enterovirus genus of the picornaviridae family (6). EV71 is classified into five genotypes as follows: A, B, C, D and F, and further subdivided into B0-B5 and C1-C5 subgenotypes (7). HFMD is an important public health issue and further understanding of the mechanisms underlying the development of HFMD are required to develop effective treatments. In mainland China, Taiwan and Singapore, EV71 whole-virus vaccines have been produced by various manufacturers. Two available inactivated EV71 vaccines have been approved by the China Food and Drug Administration, one produced by Sinovac Biotech Ltd. and the other by the Chinese Academy of Medical Science (all Beijing, China) (8-10). Currently, no effective treatment is available to treat severe EV71 infections, aside from symptomatic therapies (11).

Typically, Vero and RD cell lines have been used to isolate EV71 (12), and suckling BALB/c mice have been studied to evaluate the virulence and pathogenicity of the isolated strain (13). A previous study indicated that neonate mice were able to be fatally infected with EV71 from 0-7 days of age, whereas mice $>7$ days old hardly did not exhibit any symptoms (14). A similar situation possibly exists in adult humans, which raises various questions, including the following: Whether EV71 may be transmitted from pregnant women with occult EV71 infection to the fetuses; the pathogenic damage that occurs to the fetus when the mother is infected with EV71; and whether the neutralizing antibody for EV71 from a mother may protect her infant.

In the present study, one EV71 strain from a patient with HFMD exhibiting severe neurologic symptoms was isolated and characterized. The strain of EV71 was identified as subgenotype $\mathrm{C} 4 \mathrm{a}$, and was used as the $\mathrm{C} 4$ subgenotype has been indicated to be the most prevalent EV71 subgenotype in mainland China $(5,15)$. The present study aimed to construct an EV71-infected adult murine model to evaluate the influence of active EV71 infection in adult mice. Female adult ICR 
mice were challenged twice with isolated EV71; first by hydrodynamic injection (HI) into their tails and secondly via intraperitoneal injection (IP) as a booster. EV71 maintained a significantly higher titer with HI compared with IP challenge. Two successive high titer EV71 challenges induced mortality in the majority of the adult mice. Mice that survived were identified to have impaired fertility, and their litter size was significantly reduced compared with the control group. The antibody directed against the VP1 conserved region of EV71 persisted in female murine sera at a high titer for $>2$ years after challenge. The maternal antibody in the sera of the offspring murine also persisted for $\sim 1$ year at a moderate titer and disappeared after $\sim 2$ years.

\section{Materials and methods}

Ethics statement. Approval for the present study was obtained from the Ethics Committee of the Shaoxing People's Hospital (Shaoxing, China) and informed written consent was obtained from all patients. All animal protocols were approved by the Ethics Committee of the Institute of Animal Care of Zhejiang University (Shaoxing, China) and animals were cared for in accordance with institutional guidelines of the Shaoxing People's Hospital. Specimens were confirmed to be positive for EV71 infection by evaluating the VP1 conserved region by polymerase chain reaction (PCR) analysis (5).

Cell lines and viruses. Vero African green monkey kidney cells and RD cells were kindly provided by Professor Bo Zhang (Wuhan Institute of Virology, Chinese Academy of Sciences, Wuhan, China), and were cultured in Dulbecco's modified Eagle's medium (DMEM; Invitrogen; Thermo Fisher Scientific, Inc., Waltham, MA, USA) supplemented with 10\% fetal bovine serum (Gibco; Thermo Fisher Scientific, Inc.), $100 \mathrm{U} / \mathrm{ml}$ penicillin and $100 \mu \mathrm{g} / \mathrm{ml}$ streptomycin at $37^{\circ} \mathrm{C}$ in an atmosphere containing $5 \% \mathrm{CO}_{2}$. A total of 300 filtered swab samples from 3- to 5-year-old children (one sample per child; sex ratio was 1:1) with HFMD were collected in Shaoxing, China, between 2012 and 2013 and were added to the wells with cultured Vero cells ( 2 wells per sample, $\sim 10^{6}$ cells per well) as previously described (5,15). EV71 strains in the culture supernatant were harvested by thawing and refreezing based upon the appearance of cytopathic effects, such as pyknosis, turning round, and floating in the medium. Following propagation by cell culture and identification using the plaque assay (described below), the EV71 virus stock was adjusted to $1.0 \times 10^{7} \mathrm{pfu}$ and used as the parental virus in all assays (16).

Serum cytokine and chemokine detection. Cytokines and chemokines in the sera $(10 \mu \mathrm{l})$ from EV71-infected mice were quantitatively measured by FLEXMAP 3D quantification (Luminex Corporation, Austin, TX, USA) as described previously (17). A series of cytokines, including interleukin (IL) $1-\alpha, 1 \beta, 2,3,4,5,6,10,17 \mathrm{~F}$ and $21,12 \mathrm{p} 40,12 \mathrm{p} 70,17 \mathrm{~A}$, $13,23 \mathrm{p} 19$, interferon- $\gamma$-inducible protein 10 (IP-10), keratinocyte chemoattractant $(\mathrm{KC}) /$ chemokine C-X-X moftif ligand 1 (CXCL1), interferon $-\gamma$, granulocyte-macrophage colony-stimulating factor, and tumor necrosis factor (TNF)- $\alpha$, eotaxin and monocyte chemoattractant protein (MCP)-1, were detected using Milliplex MAP kits (cat. no. RCYTO-80K; EMD Millipore, Billerica, MA, USA), which were used in combination as described previously (17). The mean of $\geq 2$ independent measurements was calculated using xPONENT software version 3.1 (Luminex Corporation).

Plaque assay. Viruses in samples were routinely amplified by passaging the swab-derived virus through the RD cells for one round ( $24 \mathrm{~h}$ ) prior to reinfection with Vero cells for the plaque assay. Virus stock was produced by harvesting the supernatant of EV71-infected-Vero cells. Virus titer and morphology were determined via a bilayer plaque assay (16). A series of 1:10 dilutions were prepared by diluting $15 \mathrm{ml}$ virus stock with $135 \mathrm{ml}$ DMEM supplemented with $10 \%$ FBS, and $100 \mathrm{ml}$ of each dilution was seeded into each well of a 6-well plate containing confluent Vero cells $\left(1 \times 10^{6}\right.$ cells/well; plated 1 day in advance) as described previously (18). Plates were incubated at $37^{\circ} \mathrm{C}$ for $1 \mathrm{~h}$ before the first layer of $1.2 \%$ agar was added. After $72 \mathrm{~h}$ of incubation at $37^{\circ} \mathrm{C}$, a second layer of $1.2 \%$ agar containing the neutral red $\mathrm{pH}$ indicator was added. Following incubation at $37^{\circ} \mathrm{C}$ for $12-24 \mathrm{~h}$, images of the plaques were captured and the number of formed plaques was determined as described previously $(16,18)$. The viral titer was calculated as $\mathrm{pfu} / \mathrm{ml}$. The limit of detection was $10 \mathrm{pfu} / \mathrm{ml}$.

Determination of viral titers in the tissue and serum samples of infected mice. A total of 100 female ICR mice (8-10 weeks old; $20.36 \pm 1.31 \mathrm{~g}$ ) were purchased from the Model Animal Research Center of Nanjing University (Nanjing, China) and maintained under specific pathogen-free conditions $\left(22-26^{\circ} \mathrm{C}\right.$, $40-70 \%$ humidity, $10 \mathrm{~h}$ light and $14 \mathrm{~h}$ dark per day) in the Central Animal Laboratory of Shaoxing Hospital of Zhejiang University. Mice were housed in cages with a bevel allowing free access to food and water. A total of 12 mice (6 per group) were challenged with $1 \mathrm{ml}$ EV71 $\left(1 \times 10^{7} \mathrm{pfu} / \mathrm{ml}\right)$ via HI or IP. Mice were sacrificed by cervical dislocation 24 or $48 \mathrm{~h}$ post injection $(19,20)$. Blood samples were collected from the orbital vein, and organs and tissues were harvested after systemic perfusion with $50 \mathrm{ml}$ sterile PBS. The organs and tissues were weighed and then homogenized in PBS using a mechanical homogenizer, and disrupted by three freeze-thaw cycles after sacrifice, as described previously (21). Subsequently, viral titers in the supernatants of purified homogenates of the liver, brain, kidney, heart, spleen and lung, together with blood, were quantified by reverse transcription-quantitative PCR (RT-qPCR) analysis.

$R N A$ extraction and $R T-q P C R$. For RT-qPCR, viral RNA was extracted from fresh murine tissue homogenates, the blood, and throat and anal samples using TRIzol reagent (Invitrogen; Thermo Fisher Scientific, Inc.). Following digestion with DNase I, extracted RNA was reverse transcribed into cDNA using a High-Capacity cDNA Reverse Transcription kit (Applied Biosystems; Thermo Fisher Scientific, Inc.) and oligo-d(T) primers according to the manufacturer's instructions. The following primers were designed to target the VP1 conserved region of EV71: Forward, 5'-GCAGCCCAAAAG AACTTCAC-3' and reverse, 5'-ATTTCAGCAGCTTGG AGTGC-3'. RT-qPCR was performed for 40 cycles as follows: $95^{\circ} \mathrm{C}$ for $5 \mathrm{sec}, 55^{\circ} \mathrm{C}$ for $15 \mathrm{sec}$ and $72^{\circ} \mathrm{C}$ for $9 \mathrm{sec}$ using an 


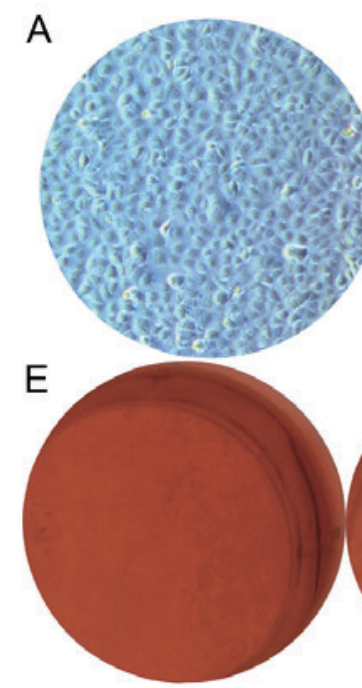

$10^{2}$

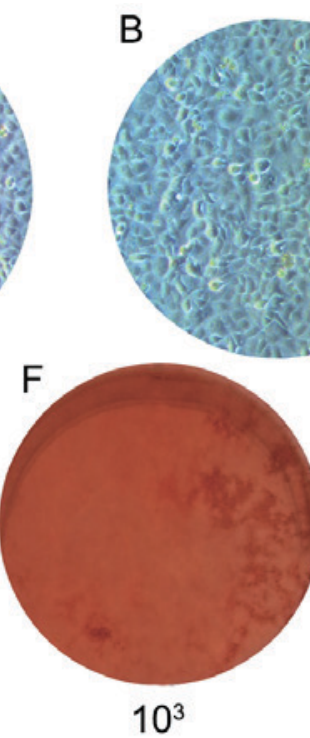

$10^{3}$

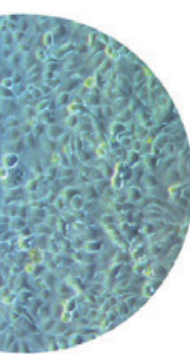

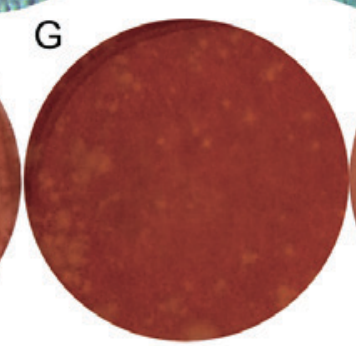

$10^{4}$
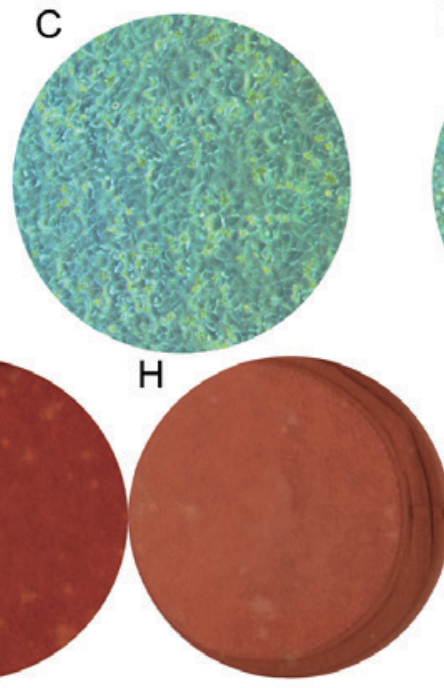

$10^{5}$
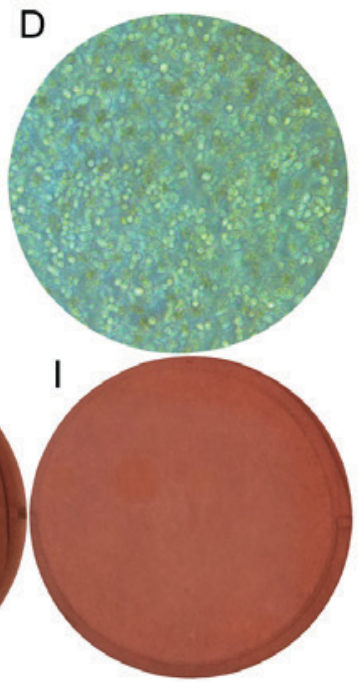

$10^{6}$

Figure 1. Viral titer and morphology of EV71 isolates from clinical specimens was determined using a bilayer plaque assay. EV71-positive samples identified by reverse-transcription-quantitative polymerase chain reaction were used to inoculate Vero cells and images were captured after (A) 24 , (B) 48 , (C) 72 and (D) $96 \mathrm{~h}$. Virus stock produced by harvesting Vero cell supernatant after characteristic cytopathic effects were observed. Virus titer and morphology were determined using a bilayer plaque assay. A series of 1:10 dilutions were administered by diluting $15 \mathrm{ml}$ virus stock with $135 \mathrm{ml}$ medium: (E) $1 \times 10^{2}$, (F) $1 \times 10^{3}$ $\left(\mathrm{G} 1 \times 10^{4}(\mathrm{H}) 1 \times 10^{5}\right.$ and (I) $1 \times 10^{6}$ dilution fold. A total of $100 \mathrm{ml}$ of each mixture was added into each well of a 6 -well plate seeded with confluent Vero cells. EV71, enterovirus 71 .

Mx3005P machine (Agilent Technologies, Inc., Santa Clara, CA, USA) as previously described $(5,15)$. The final reaction volume was $25 \mu \mathrm{l}$, including $1 \mu \mathrm{l}$ each of forward and reverse primers, $5 \mu 15 \mathrm{X}$ reaction buffer, $5 \mu 1$ sample as template, $1 \mu 1$ DNA polymerase and $12 \mu \mathrm{l}$ water. An EV71 reporter virus (GenBank accession no. JN256064; provided by Professor Bo Zhang, Wuhan Institute of Virology, Chinese Academy of Science, China) with whole EV71 sequence was used as a standard to calculate the EV71 copy number as described previously (18).

Mouse EV71 challenge. A total of $1 \mathrm{ml}$ Vero culture supernatant with isolated EV71 (1.0x $\left.10^{7} \mathrm{pfu}\right)$ was quickly injected into the tail veins of mice to maintain high pressure, or into the enterocoelia, as described previously (20). The control group (6 mice) received the same volume of DMEM. A booster injection was administered 2 weeks following the first injection. Blood samples were harvested 24 and $48 \mathrm{~h}$ after the last immunization.

For the lethalality assay, four groups of female ICR mice (6-8 weeks old, 8 mice per group) were challenged with isolated EV71 using HI or IP. Group 1: Primer and boost challenge with active EV71; group 2: Primer challenge with active EV71 and boost with inactive EV71; group 3: Primer challenge with inactive EV71 and boost with active EV71; group 4: Primer challenge with inactive EV71 and boost with inactive EV71. Survival rates were determined using GraphPad Prism software (version 5; GraphPad Prism Software, Inc., La Jolla, CA, USA).

For the reproductive damage assay, 2 groups of female mature ICR mice were challenged with active EV71 (24 mice) or DMEM as control (6 mice) by $\mathrm{HI}$ and then raised with male adult ICR mice to conceive. Booster challenge with active EV71 or DMEM was administered to the female mice via IP two weeks post primer challenge. Control group: Primer and boost challenge both with DMEM. 'Once' group: Primer challenge with active EV71 and boost with DMEM. 'Twice' group: Primer and boost challenge both with active EV71. The litter size of all surviving female mice after birth was noted and used for the assessment of reproductive ability.

For evaluation of the protectiveness of maternal antibody, 1-day-old ICR neonatal mice (weight, 1.8-2.0 g) born from the EV71-challenged and mock mice were challenged with $50 \mu 1$ active EV71 or DMEM via intracranial injection as previously described $(22,23)$. All mice were monitored for clinical symptoms, paralysis, and death for at least 14 days post-injection. In addition, for dynamic regularity of EV71-VP1-IgG, the EV71-challenged maternal mice and their second generation mice were randomly allocated into 2 groups of 10 mice, and were monitored daily for clinical signs of infection, including ruffled hair, hunched back, paralysis and mortality until the end of the assay (120 weeks), as described previously (22).

Statistical analysis. Statistical analysis was performed using GraphPad Prism software (version 5; GraphPad Prism Software, Inc.). The significance of all differences in multiple comparisons were determined using the Student's t-test. Results are presented as the mean \pm standard deviation of three independent experiments. $\mathrm{P}<0.05$ was considered to indicate a statistically significant difference.

\section{Results}

Characterization of the isolated EV71 strain. The EV71 strain used as virus stock in the present study was isolated from clinical samples from children with HFMD. The viruses infected Vero cells, inducing typical CPE as shown in Fig. 1A-D. In a previous study by the present authors, the VP1 sequence of this 

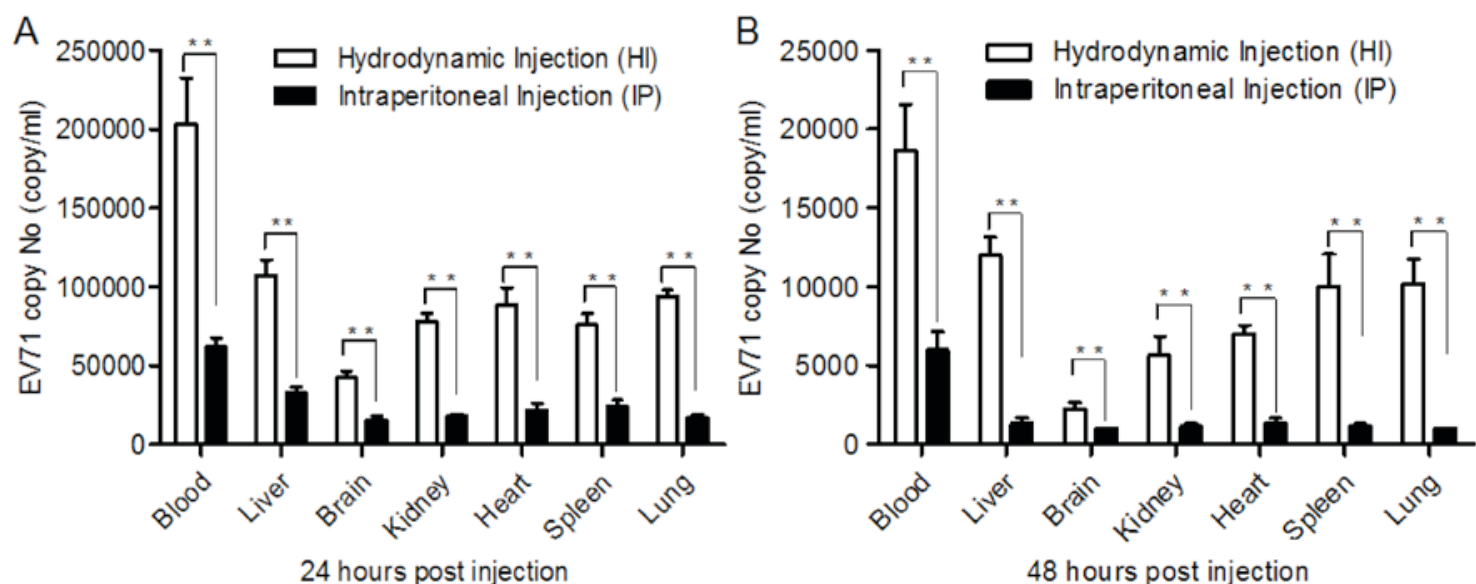

Figure 2. Determination of viral titers in blood, organ and tissue samples from EV71-infected mice. ICR female mice were challenged with active EV71 using HI or IP. Challenged mice were sacrificed (A) 24 or (B) $48 \mathrm{~h}$ post-injection. Blood was collected and different tissues were harvested. RNA extracted from the $\mathrm{b}$ samples as then subjected to reverse transcription-quantitative polymerase chain reaction analysis. ${ }^{* *} \mathrm{P}<0.01$. EV71, enterovirus 71 ; HI, hydrodynamic injection; IP, intraperitoneal injection.

A Survival of two groups:survival proportions

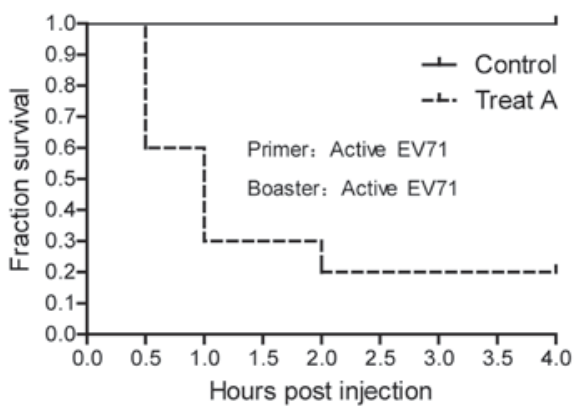

$\mathrm{D}$

Survival of two groups: survival proportions

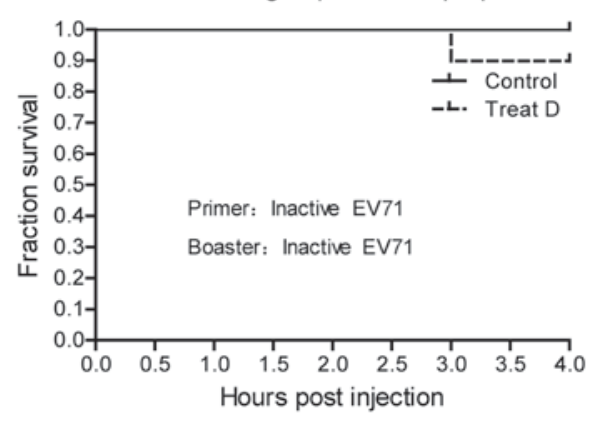

B Survival of two groups: survival proportions

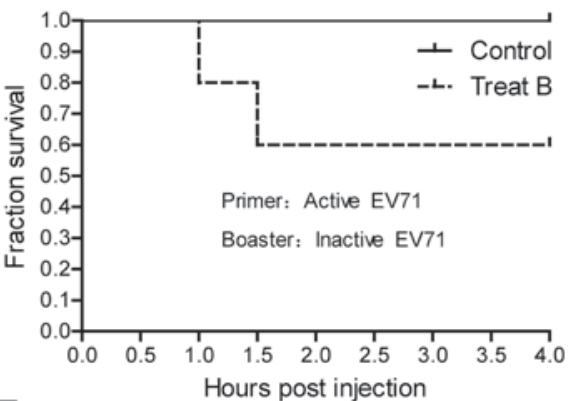

$\mathrm{E}$

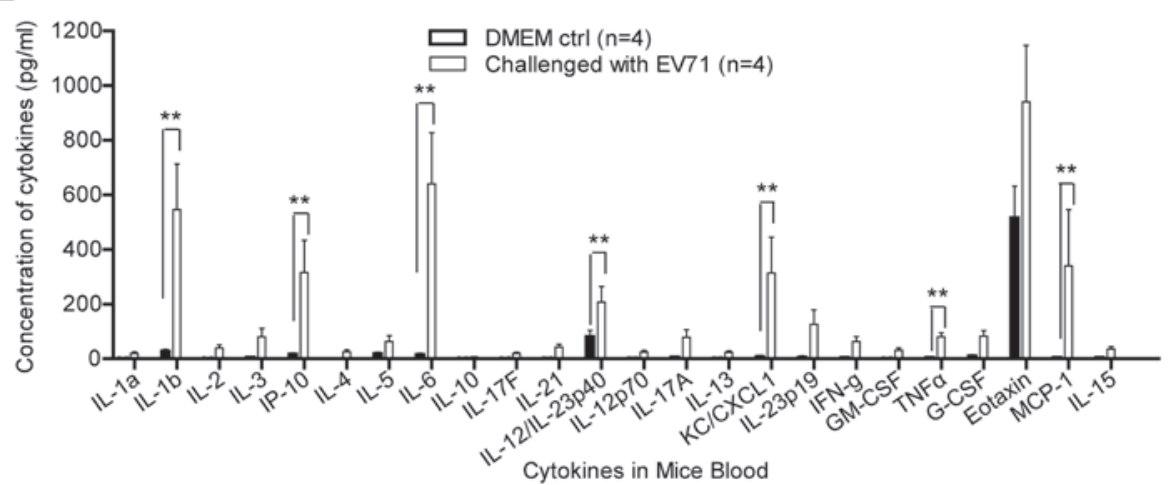

Figure 3. EV71 challenge is lethal to adult mice. Female ICR mice were divided into the following four groups: (A) active EV71 primer and booster challenge; (B) active EV71 primer and inactive EV71 booster challenge; (C) inactive EV71 primer and active EV71 booster challenge; and (D) inactive EV71 primer and booster challenge. (E) Chemokines and cytokines detected in the blood samples from mice that survived Ev71 challenge. ${ }^{* *} \mathrm{P}<0.01$ vs. the DMEM control group. EV71, enterovirus 71; DMEM, Dulbecco's Modified Eagle Medium.

isolated EV71 strain was amplified, sequenced, aligned, and was identified to be of the C4a genotype $(5,15)$.

Virus stock was produced by harvesting the supernatant of EV71-infected Vero cells. Virus titer and morphology were determined using a bilayer plaque assay (Fig. 1E-I). A total of 21 plaques were identified in the well with a $1 \times 10^{5}$ dilution (Fig. $1 \mathrm{H}$ ). The virus solution with a titer of $2.1 \times 10^{7}$ was adjusted to $1 \times 10^{7}$ and used as the parental virus in all assays.
EV71 challenge with HI produces a higher viral titer in the tissue compared with IP. In order to achieve a higher titer EV71 in the tissue of mice, administration of EV71 by HI and IP was compared. Tissue samples were homogenized in sterile PBS, disrupted by three freeze-thaw cycles and centrifuged at 14,000 x g. EV71 copy number was calculated by RT-qPCR. As indicated in Fig. 2A and B, administration via HI resulted in a significantly increased EV71 titer compared with IP at 24 and $48 \mathrm{~h}$ after injection $(\mathrm{P}<0.05)$, which indicated that $\mathrm{HI}$ 
A

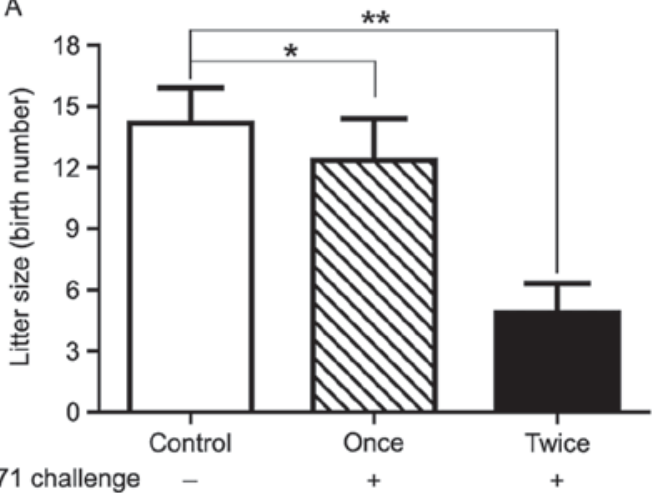

C

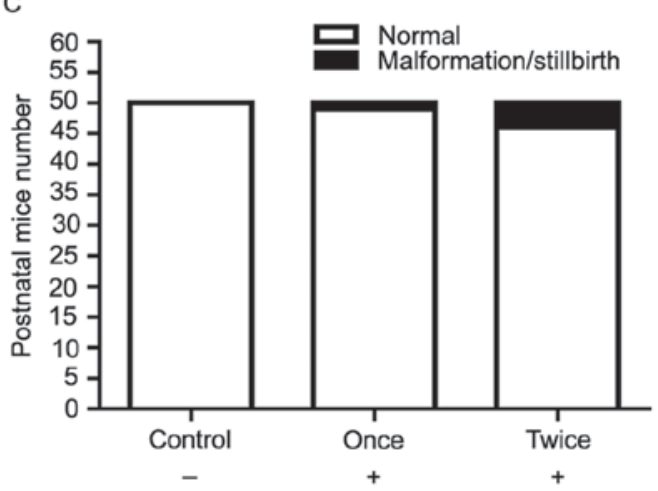

B

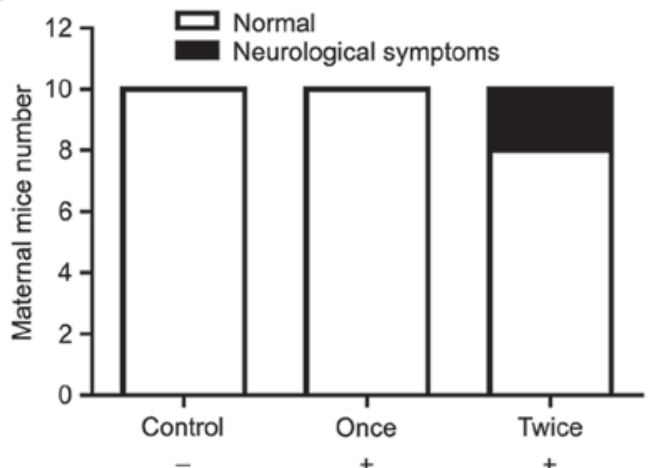

Figure 4. Reproductive capacity of female mice is impaired by EV71 infection. Female mature ICR mice were challenged with EV71 infection twice and raised with male mice to conceive. (A) EV71 challenge resulted in impaired reproductive capacity, decreasing litter size. (B) Mice challenged with EV71 exhibited neurologic symptoms. (C) Number of suckling mice born from EV71-infected mice with abnormalities. ${ }^{*} \mathrm{P}<0.05 ;{ }^{* *} \mathrm{P}<0.01$. EV71, enterovirus 71.

was preferable for acquiring a high viral titer in the tissue and blood.

EV71 challenge is fatal to adult mice. Groups of female ICR mice were challenged with isolated EV71 via HI or IP. Four $\mathrm{h}$ post booster injection, a total of $80 \%$ of mice succumbed to EV71 infection as a result of primer and booster challenge (Fig. 3A), 40\% of mice succumbed to EV71 when first challenged with active EV71 and then receiving inactive EV71 as a booster (Fig. 3B), 80\% of mice survived when first challenged with inactive EV71 and then receiving active EV71 as a booster (Fig. 3C) and 90\% of mice survived when treated with inactive EV71 as a primer and booster (Fig. 3D). It was noted that mortality was highest half an hour post booster injection.

To further investigate why EV71 challenge induced sudden fatality the blood samples from mice that survived primer and booster challenge with active EV71 virus alone were collected and subjected cytokine/chemokine detection. IL-1b, IP-10, IL-6, IL-12, KC/CXCL1, IL-23p19, TNF- $\alpha$ and MCP-1 were significantly upregulated in EV71-challenged mice compared with the non-challenged control group $(\mathrm{P}<0.01$; Fig. $3 \mathrm{E})$. These results suggest that the fatality of the challenged mice was promoted by potent immunological stress, not the infectivity and virulence of EV71 Furthermore, these findings suggest that active EV71 possesses high antigenicity compared with inactive EV71, which triggers immunoreaction.

EV71 challenge impairs the reproductive capacity of female mice. Female mature ICR mice in the 'Twice' group were challenged with active EV71 by HI before and after pregnancy. A large proportion of the mice succumbed to EV71 infection. Mice that survived were housed under specific pathogen-free conditions in individual ventilated cages until parturition. Mice in the 'Twice' group gave birth to a mean of 5 suckling mice, whereas litter size in the 'Once' group was 12 and in the control group was 14. Active EV71 administered as a primer and/or booster was identified to significantly impair the reproductivity of female mice compared with the control group $(\mathrm{P}<0.05$; Fig. 4A). A total of $20 \%$ of survivors challenged twice with EV71 exhibited neurologic symptoms, including torticollis and walking disorder (Fig. 4B). A number of neonatal mice produced from female mice challenged with active EV71 presented with abnormalities, including stillbirth and dwarfism. The proportion of abnormalities were in line with the challenge number (Fig. 4C).

EV71 antibody is maintained at a high titer for a longer duration after challenge with EV71 twice in maternal and postnatal mice. Blood samples were collected from 1-120 after the booster injection and EV71 antibody levels were detected using an ELISA kit. Maternal EV71-VP1 antibody titer reached a peak $\sim 4$ weeks after the booster injection and persisted at a high level for $\sim 1$ year, following which it slowly declined until the end of monitoring (Fig. 5A). Maternal antibodies in the blood samples taken from postnatal mice after birth were also monitored. As illustrated in Fig. 5A, the level of EV71-VP1 antibody gradually declined after birth and was barely detectable at the end of monitoring. This maternal 
A

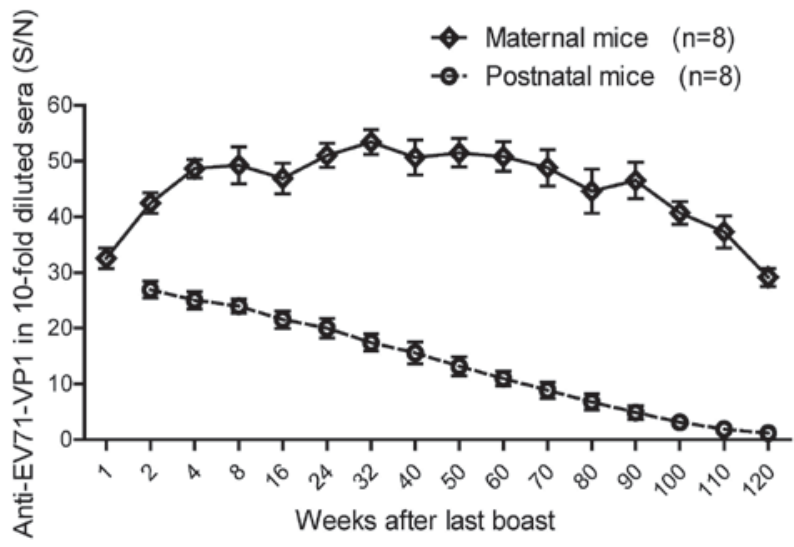

B

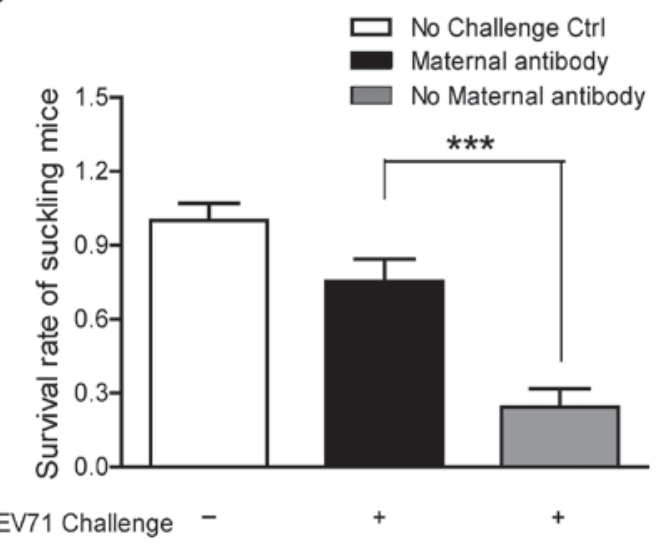

Figure 5. EV71 challenge induced a high titer of protective antibody. (A) EV71 antibody present in the sera of challenged mice and their offspring over 120 weeks. (B) Suckling mice with the maternal antibody were challenged with active EV71 by intracranial injection A higher titer of maternal antibody protected suckling mice challenged with active EV71 from mortality. ${ }^{* * * *} \mathrm{P}<0.001$. EV71, enterovirus 71 ; Ctrl, control.

antibody was indicated to be protective, as 3-day-old suckling mice with the maternal antibody were challenged with active EV71 by intracranial injection. Mice that were challenged with EV71 and exhibited the maternal antibody had a significantly increased survival rate compared with mice with no maternal antibody; $>70 \%$ of these mice were protected from EV71-induced fatality compared with $20 \%$ of mice without the maternal antibody $(\mathrm{P}<0.05$; Fig. $5 \mathrm{~B})$.

\section{Discussion}

HFMD typically occurs in children $<5$ years old, and EV71 and coxsackie virus A16 have been identified as the primary causes of HFMD (2). Serious neurological complications and fatalities are associated with EV71 (24). Due to a lack of effective treatment options, controlling EV71 epidemics has predominantly relied upon the formation of EV71 vaccines (11). Therefore, effective vaccines that protect against EV71-associated HFMD or herpangina are important (25). Currently, two monovalent EV71 vaccines have been marketed in mainland China (2). However, clinical data concerning their effectiveness is not yet available.

Despite the extensive research undertaken since the discovery of EV71, the following issues require further investigation: The potential harm to a pregnant woman with EV71 latent infection and her fetus; and the length of time that anti-EV71 antibody remains effective after vaccination. In the present study, an isolated C4a EV71 strain was used to challenge ICR mice. The results indicated that EV71 was maintained a higher viral titer (calculated by RT-qPCR) in murine tissue and blood when challenged via HI as displayed in Fig. 2A. Furthermore, $>50 \%$ of the challenged mice succumbed to EV71 infection when challenged with EV71 infection twice. In addition, abnormal symptoms were demonstrated in the survivors and their progenies. Based on the serum chemokine/cytokine detection performed, potent immunological stress was likely responsible for this lethality and teratogenicity, not the infectivity and virulence of EV71. Active EV71 challenge resulted in more serious lethality and teratogenicity compared with inactive EV71 challenge. However, the mechanisms underlying this effect of EV71 require further research. Furthermore, the neutralizing antibody in maternal mice and their offspring was present over a long period of time at a high titer when the mice were challenged twice. These results indicate that EV71 exhibits notable immunogenicity, suggesting its suitability as a potential vaccine candidate. In addition, the presence of the maternal antibody in the fetus was revealed to be protective against EV71-induced mortality, which suggests that children $<5$ years old and women of child-bearing age may benefit from receiving EV71 vaccination.

The majority of previous studies have reported that adult mice are not susceptible to EV71 infection, with only suckling mice able to be infected by EV71. To the best of our knowledge, the present study is the first to report that active EV71 challenge inhibits the reproductive capacity of female mice, and induces neurologic symptoms in adults and offspring. In humans, occult EV71 infection is common in adults without any symptoms (2), who may act as a reservoir host for susceptible infants. Furthermore, if a woman of childbearing age is infected with EV71, little is known about whether this infection would harm to her fetus. Thus, further studies warranted to investigate this effect of EV71 in humans.

In conclusion, a novel challenge method of EV71, hydrodynamic injection, was established in the present study. Due to the higher titer of EV71 in the tissue of challenged mice, it was demonstrated to be more effective than intraperitoneal injection. Generally speaking, EV71 infection was only fatal to suckling mice within 7 days of birth. In the present study, a higher rate of teratogenesis occurred in adult mice following EV71 infection. In particular, the twice-active EV71 challenge may cause mortality in adult mice, and surviving mice have a significantly lower reproductive capacity. These results suggest that occult EV71 infection in humans, in particular women of childbearing age, may pose a serious health risk.

\section{Acknowledgements}

The present study was supported in part by the Medical Scientific Research Foundation of Zhejiang Province 
(grant no.2013KYA208) and the Science Technology Bureau of Shaoxing, Zhejiang province of China (grant nos. 2013B70063 and 2012B70063).

\section{References}

1. Ye X, Fan C, Ku Z, Zuo T, Kong L, Zhang C, Shi J, Liu Q, Chen T, Zhang Y, et al: Structural basis for recognition of human enterovirus 71 by a bivalent broadly neutralizing monoclonal antibody. PLoS Pathog 12: e1005454, 2016.

2. Gui J, Liu Z, Zhang T, Hua Q, Jiang Z, Chen B, Gu H, Lv H and Dong C: Epidemiological characteristics and spatial-temporal clusters of hand, foot and mouth disease in zhejiang province, China, 2008-2012. PLoS One 10: e0139109, 2015.

3. Ho M, Chen ER, Hsu KH, Twu SJ, Chen KT, Tsai SF, Wang JR and Shih SR: An epidemic of enterovirus 71 infection in Taiwan. Taiwan Enterovirus Epidemic Working Group. N Engl J Med 341: 929-935, 1999.

4. Xu W, Liu CF, Yan L, Li JJ, Wang LJ, Qi Y, Cheng RB and Xiong XY: Distribution of enteroviruses in hospitalized children with hand, foot and mouth disease and relationship between pathogens and nervous system complications. Virol J 9: 8, 2012.

5. Qin B, Zhang J, Xie W, Liu X, He T, Chen J and Dong X: Development of a specific latex agglutination test to detect antibodies of enterovirus 71. Arch Med Res 46: 580-587, 2015.

6. Qing J, Wang Y, Sun Y, Huang J, Yan W, Wang J, Su D, Ni C, Li J, Rao Z, et al: Cyclophilin A associates with enterovirus-71 virus capsid and plays an essential role in viral infection as an uncoating regulator. PLoS Pathog 10: e1004422, 2014.

7. Liu CC, Chow YH, Chong P and Klein M: Prospect and challenges for the development of multivalent vaccines against hand, foot and mouth diseases. Vaccine 32: 6177-6182, 2014.

8. Liang ZL, Mao QY, Wang YP, Zhu FC, Li JX, Yao X, Gao F, Wu X, Xu M and Wang JZ: Progress on the research and development of inactivated EV71 whole-virus vaccines. Hum Vaccin Immunother 9: 1701-1705, 2013.

9. Ren J, Wang X, Zhu L, Hu Z, Gao Q, Yang P, Li X, Wang J, Shen X, Fry EE, et al: Structures of Coxsackievirus A16 capsids with native antigenicity: Implications for particle expansion, receptor binding and immunogenicity. J Virology 89: 10500-10511, 2015.

10. Chang J, Li J, Wei W, Liu X, Liu G, Yang J, Zhang W and Yu XF: Determinants of EV71 immunogenicity and protection against lethal challenge in a mouse model. Immunol Res 62: 306-315, 2015.

11. Ng Q, He F and Kwang J: Recent Progress towards Novel EV71 anti-therapeutics and vaccines. Viruses 7: 6441-6457, 2015.

12. Li X, Fan P, Jin J, Su W, An D, Xu L, Sun S, Zhang Y, Meng X, Gao F, et al: Establishment of cell lines with increased susceptibility to EV71/CA16 by stable overexpression of SCARB2. Virol J 10: 250, 2013.
13. Wang YF, Chou CT, Lei HY, Liu CC, Wang SM, Yan JJ, Su IJ, Wang JR, Yeh TM, Chen SH and Yu CK: A mouse-adapted enterovirus 71 strain causes neurological disease in mice after oral infection. J Virol 78: 7916-7924, 2004.

14. Shih SR, Li YS, Chiou CC, Suen PC, Lin TY, Chang LY, Huang YC, Tsao KC, Ning HC, Wu TZ and Chan EC: Expression of capsid [correction of caspid] protein VP1 for use as antigen for the diagnosis of enterovirus 71 infection. J Med Virol 61: 228-234, 2000

15. Bo HTtCJkZJhJZjHXnFLjQ: A study on genetic characteristics of Enterovirus 71 of hand-foot-mouth disease in Shaoxing city Zhejiang Prev Med 26: 4, 2014.

16. Li XD, Li XF, Ye HQ, Deng CL, Ye Q, Shan C, Shang BD, $\mathrm{Xu}$ LL, Li SH, Cao SB, et al: Recovery of a chemically synthesized Japanese encephalitis virus reveals two critical adaptive mutations in NS2B and NS4A. J Gen Virol 95: 806-815, 2014.

17. Chen J, Zhao Y, Zhang C, Chen H, Feng J, Chi X, Pan Y, Du J, Guo M, Cao $\mathrm{H}$, et al: Persistent hepatitis $\mathrm{C}$ virus infections and hepatopathological manifestations in immune-competent humanized mice. Cell Res 24: 1050-1066, 2014.

18. Shang B, Deng C, Ye H, Xu W, Yuan Z, Shi PY and Zhang B: Development and characterization of a stable eGFP enterovirus 71 for antiviral screening. Antiviral Res 97: 198-205, 2013.

19. Qin B, Tu C, Zhang B, He T, Fu L and Xu W: A modified murine model based on hydrodynamic injection for the analysis of chronic human hepatitis B virus infection. Mol Med Rep 8: 1677-1682, 2013.

20. Qin B, Budeus B, Cao L, Wu C, Wang Y, Zhang X, Rayner S, Hoffmann D, Lu M and Chen X: The amino acid substitutions $\mathrm{rtP} 177 \mathrm{G}$ and $\mathrm{rtF} 249 \mathrm{~A}$ in the reverse transcriptase domain of hepatitis B virus polymerase reduce the susceptibility to tenofovir. Antiviral Res 97: 93-100, 2013.

21. Chen IC, Wang SM, Yu CK and Liu CC: Subneutralizing antibodies to enterovirus 71 induce antibody-dependent enhancement of infection in newborn mice. Med Microbiol Immunol 202: 259-265, 2013

22. Li P, Yue YY, Song NN, Li ZH and Meng H: Study on BALB/c mice with EV71 infection by diverse inoculation routes. Zhonghua Shi Yan He Lin Chuang Bing Du Xue Za Zhi 27: 14-17, 2013 (In Chinese).

23. Han JF, Yu N, Pan YX, He SJ, Xu LJ, Cao RY, Li YX, Zhu SY, Zhang Y and Qin ED: Phenotypic and genomic characterization of human coxsackievirus A16 strains with distinct virulence in mice. Virus Res 179: 212-219, 2014.

24. Teng S, Wei Y, Zhao SY, Lin XY, Shao QM and Wang J: Intestinal detoxification time of hand-foot-and-mouth disease in children with EV71 infection and the related factors. World J Pediatr 11: 380-385, 2015.

25. Klein MH: EV71 vaccines: A first step towards multivalent hand, foot and mouth disease vaccines. Expert Rev Vaccines 14: 337-340, 2015. 